\title{
Extrasynaptic Localization of Inactivating Calcium-Activated Potassium Channels in Mouse Inner Hair Cells
}

\author{
Sonja J. Pyott, ${ }^{1}$ Elisabeth Glowatzki, ${ }^{2}$ James S. Trimmer, ${ }^{3}$ and Richard W. Aldrich ${ }^{1}$ \\ ${ }^{1}$ Department of Molecular and Cellular Physiology, Howard Hughes Medical Institute, Stanford University School of Medicine, Stanford, California 94305- \\ 5345, ${ }^{2}$ Department of Otolaryngology Head and Neck Surgery, The Johns Hopkins School of Medicine, Baltimore, Maryland 21205-2195, and ${ }^{3}$ Department \\ of Pharmacology, School of Medicine, University of California, Davis, California 95616-8635
}

\begin{abstract}
Auditory hair cells from nonmammalian vertebrates are electrically tuned to specific sound frequencies primarily by the interactions of voltage-gated calcium channels and calcium-activated potassium (BK) channels colocalized at synaptic active zones. Mammalian inner hair cells are not electrically tuned and, yet, BK channels are also thought to reside at active zones. Using patch-clamp recordings and immunofluorescence, we characterized BK channel expression in mouse inner hair cells. Unexpectedly, these channels have inactivating currents and are clustered near the apex of the cell away from synaptic sites near the base. These results indicate a novel function of BK channels in mammalian inner hair cells and provide a framework for future research.
\end{abstract}

Key words: slo; KCNMA1; $\mathrm{K}_{\mathrm{Ca}} 1.1$; hearing; cochlea; synapse

\section{Introduction}

Hair cells of the vertebrate cochlea are responsible for transducing various aspects of sound. In nonmammalian vertebrates, hair cells of the cochlea are tuned to specific sound frequencies by an electrical resonance. This electrical tuning is mediated primarily by the interplay between voltage-gated calcium channels and calcium-activated potassium (BK) channels colocalized at presynaptic active zones (Fettiplace and Fuchs, 1999). Mammalian inner hair cells (IHCs), the subset of hair cells responsible for transducing sound, express a potassium conductance $\left(I_{\mathrm{K}, \mathrm{f}}\right)$ distinguished from other outward currents by its fast rate of activation (Kros and Crawford, 1990). Evidence from electrophysiology in mouse (Kros et al., 1998) and single-cell reverse transcription-PCR and in situ hybridization in rat (Langer et al., 2003) suggests that BK channels may mediate this conductance. The appearance of this conductance at the onset of hearing (Kros et al., 1998) and the delayed appearance of this conductance in thyroid hormone receptor $\beta$ (TR $\beta$ )-deficient mice (Rüsch et al., 2001 ) suggest that the acquisition of $I_{\mathrm{K}, \mathrm{f}}$ is important for the proper development of the auditory pathway. However, because mammalian IHCs are not frequency tuned by an electrical resonance as seen in nonmammalian hair cells, the precise role of BK

Received Aug. 2, 2004; revised Aug. 26, 2004; accepted Aug. $27,2004$.

This work was supported by the National Institute on Deafness and Other Communication Disorders (Grant DC006476 to E.G.), the National Institutes of Health (Grant NS34383 to J.S.T.), the National Science Foundation (graduate research fellowship to S.J.P.), and the Mathers Foundation (R.W.A.). R.W.A. is an investigator with the Howard Hughes Medical Institute. We thank P. A. Fuchs for providing the technical training required to begin these experiments and for critically reviewing this manuscript. A. Fodor, W. Li, A. Meredith, R. Piskorowski, and M. van Tuinen also gave invaluable comments on this manuscript. We also acknowledge the technical assistance of $\mathrm{L}$. Buchwalder for production of the monoclonal BK channel antibodies; K. Rhodes for help screening the monoclonal antibodies; S. Smith, M. Emond, and J. Tsui for help with confocal microscopy; and J. Nelson and S. Banhamyar for help with the three-dimensional reconstructions.

Correspondence should be addressed to Richard W. Aldrich at the above address. E-mail: raldrich@stanford.edu. D0I:10.1523/JNEUROSCI.3162-04.2004

Copyright $\odot 2004$ Society for Neuroscience $\quad 0270-6474 / 04 / 249469-06 \$ 15.00 / 0$ channels in IHCs is unknown. The general assumption has been that the BK channels also colocalize with voltage-gated calcium channels at presynaptic active zones to modulate transmitter release onto the afferent fibers (Fuchs et al., 2003; Skinner et al., 2003).

To study the properties and localization of BK channels in IHCs and their relationship to $I_{\mathrm{K}, \mathrm{f}}$, we used iberiotoxin (IBTX), a toxin known to block BK channels selectively (Galvez et al., 1990), to isolate a rapidly activating component from whole-cell currents recorded electrophysiologically from mouse IHCs (mIHCs). Additionally, we used immunofluorescence with antibodies generated against the BK channel to verify their presence in mIHCs. This combined approach shows that, indeed, BK channels mediate $I_{\mathrm{K}, \mathrm{f}}$ and that their expression is developmentally regulated. Three-dimensional reconstructions of immunostained mIHCs reveal an unexpected subcellular localization of the BK channels: surprisingly, the channels are clustered apically on the inner hair cells and away from basally terminating afferent fibers.

\section{Materials and Methods}

Electrophysiology. Apical turns of the organ of Corti were excised from C57BL/6 mice at the indicated ages and used within $1 \mathrm{hr}$ of the dissection. IHCs were identified in the turn by their morphology using a $40 \times$ water immersion objective and were accessed electrophysiologically by moving the recording electrode through the preparation under positive pressure. Extracellular solution contained the following (in $\mathrm{mM}$ ): $155 \mathrm{NaCl}, 5.8$ $\mathrm{KCl}, 0.9 \mathrm{MgCl}_{2}, 1.3 \mathrm{CaCl}_{2}, 0.7 \mathrm{NaH}_{2} \mathrm{PO}_{4}, 5.6$ D-glucose, and $10 \mathrm{HEPES}$, $\mathrm{pH}$ 7.4. For indicated experiments, $100 \mathrm{~nm}$ IBTX or $10 \mu \mathrm{M}$ paxilline (Tocris Cookson, Bristol, UK) were added to the extracellular solution and delivered locally via a gravity flow pipette made from a MicroFil needle with $250 \mu \mathrm{m}$ inner diameter (World Precision Instruments, Sarasota, FL) placed $\sim 300 \mu \mathrm{m}$ from the recording electrode. Pipette solution contained the following (in mM): $150 \mathrm{KCl}, 3.5 \mathrm{MgCl}_{2}, 5$ EGTA, 5 HEPES, and $2.5 \mathrm{Na}_{2} \mathrm{ATP}$ (ATP), pH 7.2. Recording electrodes were pulled from 
micropipettes (VWR Scientific, West Chester, PA) and coated with wax (Kerr sticky wax, Romulus, MI) to minimize electrode capacitance. Pipette resistances in solution were 2-3 M $\Omega$. Series resistances (typically 5 $\mathrm{M} \Omega$ ) were compensated $70 \%$ on-line. The indicated holding potentials are not corrected for liquid junction potentials or for the voltage drop across the remaining uncompensated series resistance. Leak currents were subtracted using a standard $\mathrm{P} / 4$ protocol (Armstrong and Bezanilla, 1977). Experiments were done at room temperature. Data were acquired with an Axopatch 200B amplifier (Axon Instruments, Foster City, CA), filtered at $10 \mathrm{kHz}$, and digitized at $50 \mathrm{kHz}$ with a 16 bit analog-to-digital converter (InstruTech ITC-16; InstruTech, Port Washington, NY) under control of Pulse acquisition software. All traces shown and used for analyses are averaged from at least five recordings. Analyses were performed with Igor (Wavemetrics, Lake Oswego, OR) and Microsoft Excel (Microsoft, Seattle, WA).

Generation of monoclonal BK channel antibodies. L6 monoclonal antibodies against the BK channel antibody were made using the GST-mslo antigen corresponding to amino acids 682-1196 of mslo (MBR5) as described previously (Bekele-Arcuri et al., 1996). The isotype of the L6/60 monoclonal antibody used in these experiments was determined as $\mathrm{IgG}_{2 \mathrm{~A}}$, and the binding site mapped to amino acids $682-859$.

Immunostaining. Whole cochleae were dissected from C57BL/6 mice of various ages and immediately perfused through the round window with $4 \%$ paraformaldehyde (PFA) in PBS, $\mathrm{pH}$ 7.4. Cochleae were allowed to fix in $4 \%$ PFA/PBS for $2-3 \mathrm{hr}$ at $4^{\circ} \mathrm{C}$ before being rinsed with PBS. Apical turns of the organs of Corti were excised from the cochleae and blocked in blocking buffer (PBS with 5\% normal goat serum and $0.2 \%$ Triton X-100) for $2 \mathrm{hr}$ at room temperature. Turns were incubated in the primary antibody diluted in blocking buffer overnight at $4^{\circ} \mathrm{C}$, rinsed three times for $20 \mathrm{~min}$ in PBT (PBS with $0.2 \%$ Triton X-100), incubated in the secondary antibody diluted in blocking buffer for $4 \mathrm{hr}$ at room temperature, rinsed three times for $20 \mathrm{~min}$ in PBT, and rinsed in PBS before mounting on glass slides in Vectashield mounting medium (Vector Laboratories, Burlingame, CA). All incubations and rinses were performed on a rocking table.

Preabsorption controls were performed by incubating a concentrated $(\sim 10 \times)$ solution of the BK channel and calretinin antibodies with the GST-mslo fusion protein for $2 \mathrm{hr}$ at $37^{\circ} \mathrm{C}$. Solutions were then spun at $15,000 \times g$ for 20 min before diluting to the final working concentration. Immunostaining was performed as described above. For preabsorption controls of the monoclonal BK channel antibody, $400 \mu \mathrm{g} / \mathrm{ml}$ of the GSTmslo antigen was used. For preabsorption controls of the polyclonal BK channel antibody, $10 \mu \mathrm{g} / \mathrm{ml}$ of the commercially provided fusion protein was used.

The monoclonal antibody against the BK channel was used at a final concentration of $10 \mu \mathrm{g} / \mathrm{ml}$. The polyclonal antibody against the BK channel was purchased from Alomone Labs (Jerusalem, Israel) and diluted 1:500. Monoclonal and polyclonal antibodies against calretinin were purchased from Chemicon (Temecula, CA) and diluted 1:1000. The monoclonal antibody against neurofilament-200 was graciously provided by Novocastra Laboratories (Newcastle upon Tyne, UK) and diluted 1:50. All secondary antibodies (Alexa Fluor 488, 594, and 647) were purchased from Molecular Probes (Eugene, OR) and diluted 1:1000.

Microscopy. Simultaneous one- or two-color fluorescence images were acquired on a laser scanning confocal microscope (designed by Stephen J. Smith, Stanford University, Stanford, CA) with a Zeiss (Thornwood, NY) $63 \times$ or $40 \times$ oil immersion Fluar objective [numerical aperture (NA), 1.3]. Developmental immunofluorescence images were quantified for number of puncta using NIH ImageJ software. Specifically, images were equivalently thresholded, converted to binary (black and white) images, and analyzed for the number of particles of sizes between 5 and 10,000 pixels for equal fields of view $(86.4 \times 115.2 \mu \mathrm{m})$. An average value for a specific developmental time point from a single experiment was taken from three to six micrographs. These values were then averaged across three independent experiments.

Z-stacks of three-color fluorescence images were acquired on a Zeiss LSM 510 laser scanning confocal microscope with a $40 \times$ oil immersion Neofluor objective (NA, 1.3). The row of IHCs was placed in the center of the field, and a z-stack (30-40 optical sections) was collected at $\sim 0.4 \mu \mathrm{m}$
P10

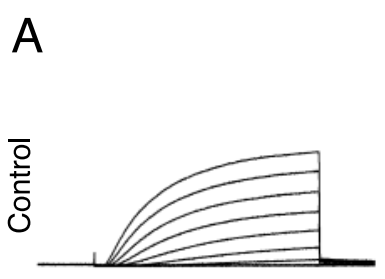

B

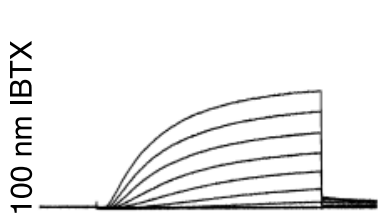

C
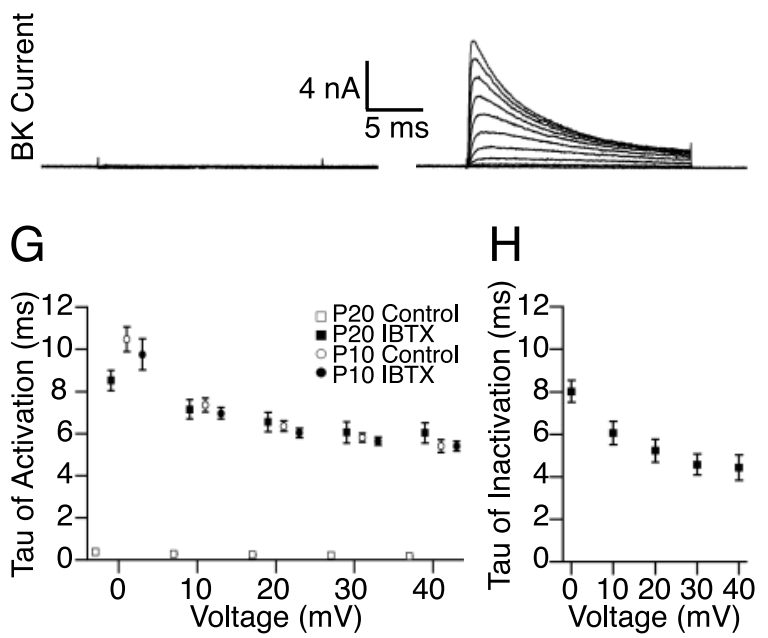

Figure 1. IBTX isolates a fast activating and inactivating outward current from IHCs of mice with mature hearing. Outward currents in response to depolarizations from -60 to $40 \mathrm{mV}$ in 10 $\mathrm{mV}$ steps were recorded from IHCs from intact mouse organs of Corti as described in Materials and Methods. $A, B$, Outward currents recorded from IHCs from P10 mice activate slowly $(A)$ and are not affected by the addition of $100 \mathrm{~nm}$ IBTX in the extracellular solution $(B) . D, E$, In contrast, outward currents recorded from IHCs from P20 mice show a rapidly activating component (D) that is blocked by the presence of $100 \mathrm{~nm} \operatorname{IBTX}(E)$. C, F, Subtraction of the currents recorded in the presence of IBTX from the total currents recorded in control solution reveals little BK current in IHCs from P10 mice $(C)$ and the presence of an inactivating BK current in IHCs from P2O mice ( $F$. $G$, The time constants of activation for these currents are compared over a range of positive command potentials. $H$, The voltage dependence of inactivation of the BK current isolated from IHCs of P2O mice is shown over the same voltage range.

intervals to capture the entirety of the cell bodies. Optical sections were combined, and three-dimensional reconstructions were performed through volume rendering using Volocity 2.0.1 (Improvision, Lexington, MA). The $0^{\circ}$ angles were set arbitrarily.

Statistics. Values are reported as the mean \pm SEM.

\section{Results}

\section{IHCs from mice with mature hearing have fast activating and} inactivating BK currents

Because the molecular identities of many of the channels expressed in IHCs are unknown, we used IBTX to isolate a BK current from whole-cell currents recorded from IHCs in acutely excised mouse organs of Corti. IHCs were voltage clamped, and outward currents were recorded in response to depolarizations from a holding potential of -60 to $40 \mathrm{mV}$ in $10 \mathrm{mV}$ steps (Fig. 1). Before the onset of hearing at postnatal day 12 (P12) (Shnerson 
and Pujol, 1981), outward membrane currents rise slowly (Fig. $1 A)$. Application of $100 \mathrm{~nm}$ IBTX ( $\mathrm{IC}_{50}<10 \mathrm{~nm}$ for mIHCs) (Kros et al., 1998) abolishes little of this outward current (Fig. 1B) and fails to affect the time constant of activation over a range of command voltages ( $p=0.9$ at $40 \mathrm{mV} ; n=5$ ) (Fig. $1 G$ ). Subtraction of the currents recorded in the presence of IBTX from traces recorded in control solution reveals minimal IBTX-sensitive current (Fig. 1C). In contrast, outward currents recorded from mIHCs with mature hearing (P20) (Shnerson and Pujol, 1981) show an additional fast component $(\tau, 0.15 \pm 0.02 \mathrm{msec}$ at 40 $\mathrm{mV}$ ) (Fig. 1D) previously documented (Kros et al., 1998). Application of IBTX abolishes this fast component (Fig. 1E). The remaining IBTX-insensitive current (Fig. $1 E$ ) shows activation rates $(\tau, 6.0 \pm 0.5 \mathrm{msec}$ at $40 \mathrm{mV})$ comparable with the activation rates of currents recorded from $\mathrm{P} 10$ preparations before $(\tau, 5.4 \pm$ $0.3 \mathrm{msec}$ at $40 \mathrm{mV})$ and after $(\tau, 5.4 \pm 0.2 \mathrm{msec}$ at $40 \mathrm{mV})$ application of IBTX $(p=0.2$ at $40 \mathrm{mV})$. These data are summarized across a range of positive voltages and conditions in Figure $1 G$.

The known specificity of IBTX for BK channels combined with the IBTX sensitivity of only the quickly activating component of outward current show that BK channels mediate $I_{\mathrm{K}, \mathrm{f}}$ in mIHCs. Moreover, these data demonstrate the voltage dependence of the activation and inactivation kinetics for the BK current in this preparation. Strikingly, isolation of the IBTXsensitive current shows that this current inactivates (Fig. $1 F, H$ ). We also isolated similar inactivating BK currents using paxilline, an additional BK channel-specific peptide blocker (Sanchez and McManus, 1996). Previous attempts to isolate the BK current in mIHCs using toxins to block other components of the outward current described a current that did not inactivate (Oliver et al., 2003). However, given the unknown molecular identity of the channels contributing to the outward current in mIHCs and the lack of specificity of the blockers used in that study, the results may have been confounded by inadequate isolation of the BK component. More recent work using IBTX subtraction to isolate a BK current from mIHCs also reported an inactivating current that became noninactivating after applying an off-line procedure for correcting the residual series resistance error (Marcotti et al., 2004). However, residual series resistance error should underestimate the amount of inactivation seen in subtraction because peak currents in the subtracted trace would be artifactually reduced more than the steady state currents. In line with this reasoning, in our data, the amount of current (which is proportional to the series resistance error) was correlated to a decrease in the rate of inactivation measured (data not shown). Although the residual series resistance error cannot be responsible for the apparent inactivation, the specific values for the inactivation rates may be underestimates. Marcotti et al. (2004) also examined currents over a more negative voltage range, a range in which inactivation is less apparent. Inactivation of the BK current has been observed in both nonmammalian hair cells (Armstrong and Roberts, 2001) and mammalian IHCs (Kimitsuki et al., 2003; Skinner et al., 2003).

\section{BK channels are clustered in puncta on mouse IHCs}

Using an independent method to verify the presence of BK channels in mIHCs, we immunostained excised organs of Corti from mice with mature hearing (P20) with both monoclonal and polyclonal antibodies generated against the BK channel. The organization of the organ of Corti into one row of IHCs and three rows of outer hair cells is shown by immunostaining against calretinin (green), an endogenous calcium binding protein enriched in the cytosol of hair cells (Zheng and Gao, 1997). BK channel immu-
A

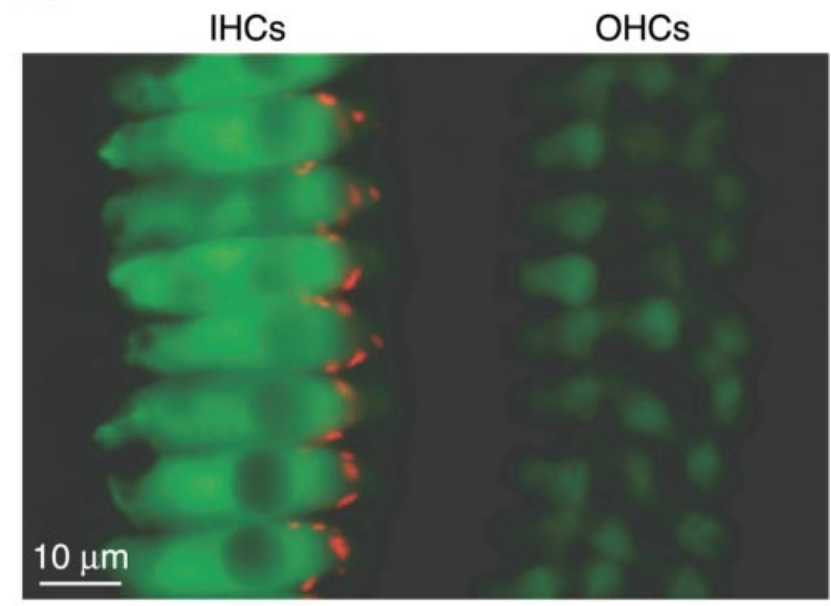

B
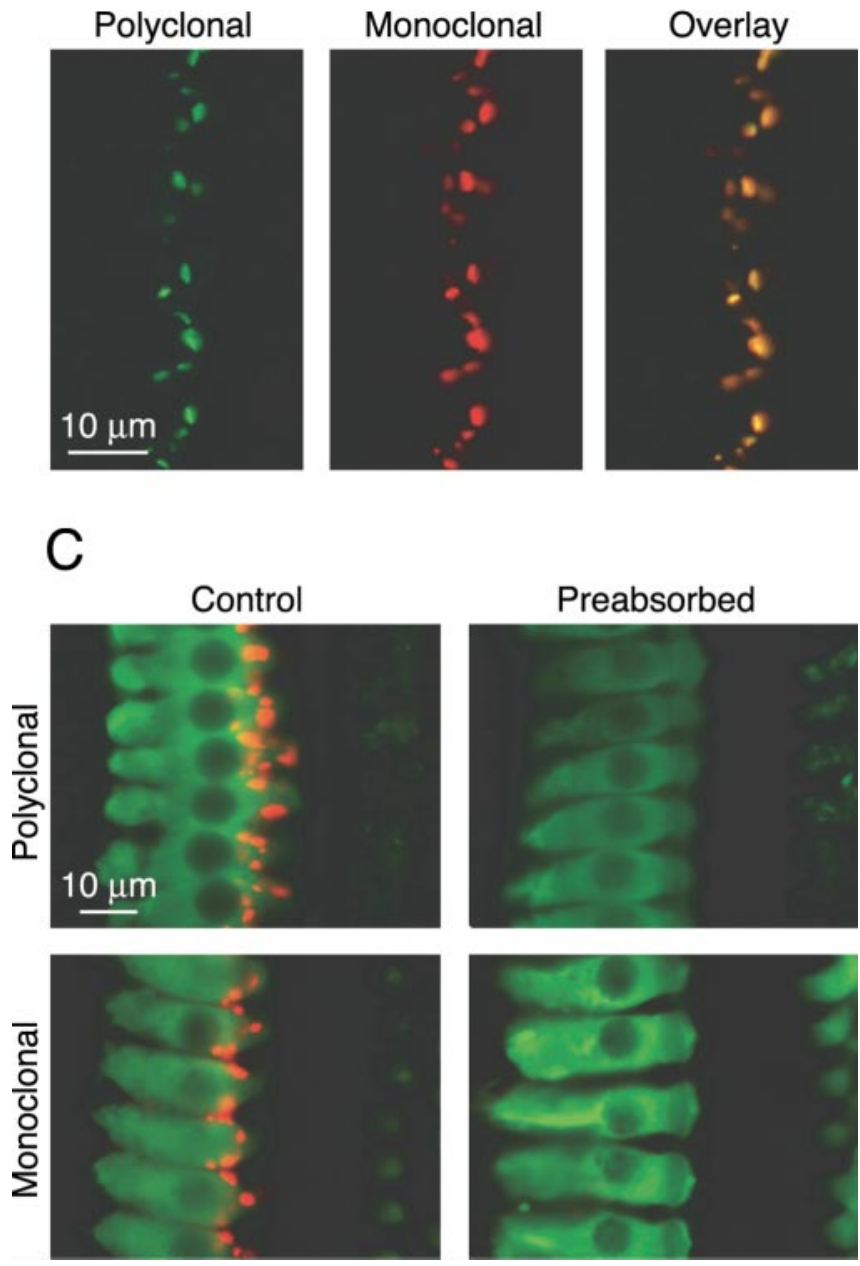

Figure 2. Antibody staining against the BK channel reveals punctate staining on $\mathrm{mIHCs}$. Intact organs of Corti from P20 mice were immunostained as described in Materials and Methods. $A$, Immunostaining with a monoclonal calretinin antibody (green) and polyclonal BK channel antibody (red) shows the organization of the hair cells into three rows of outer hair cells and one row of IHCs that additionally shows punctate staining for the BK channel. $B$, The monoclonal BK channel antibody recognizes the same protein as the polyclonal antibody as evidenced by colocalized immunoreactivity in samples immunostained with both the polyclonal (green) and monoclonal (red) BK channel antibodies. C, Preabsorption of either the polyclonal or monoclonal BK channel antibody abolishes immunoreactivity on the IHCS. 
noreactivity (red) is clustered in puncta on the IHCs (Fig. 2A). The specificity of this staining is confirmed by the similar patterns of punctate staining observed on the IHCs when staining with either a monoclonal or polyclonal BK channel antibody (Fig. 2 B, C). Each antibody recognizes discrete portions of the intracellular $\mathrm{C}$ terminus of the channel: the polyclonal was generated against amino acids 1098-1196, and the monoclonal recognizes an epitope between amino acids 682 and 859. Double immunostaining with the polyclonal and monoclonal antibody in the same sample yield colocalized puncta (Fig. 2B), suggesting they are recognizing the same protein. Moreover, preabsorption of either antibody with the protein used to generate the antibody abolishes the punctate staining observed in the IHCs (Fig. 2C), whereas preabsorption with a protein for another voltage-gated potassium channel $\left(\mathrm{K}_{\mathrm{V}} 1.4\right)$ has no effect on staining (data not shown). Together with previous work demonstrating the presence of BK channel mRNAs in mammalian IHCs (Langer et al., 2003), our findings suggest that the BK channel immunoreactivity is intrinsic to the IHCs and not coming from closely neighboring structures.

\section{BK current and BK channel}

immunoreactivity in mouse IHCs show similar developmental increases

To determine whether the BK channels detected by immunofluorescence correlate to the $\mathrm{BK}$ currents detected electrophysiologically from mIHCs, we compared the developmental acquisition of the BK current to the developmental acquisition of BK channel immunoreactiv-

ity. Figure $3 A$ shows representative traces of whole-cell BK currents isolated (as described in Fig. 1) from mIHCs before the onset (P10), just after the onset (P14), and after the maturation (P20) of hearing. These data are quantified across multiple cells as a function of the command potential (Fig. $3 C$ ) and clearly show the developmental acquisition of a BK current in mIHCs at the onset of hearing, paralleling the acquisition of $I_{\mathrm{K}, \mathrm{f}}$ reported previously (Kros et al., 1998). Specifically, at a command potential of $40 \mathrm{mV}$, the IBTX-sensitive current isolated from IHCs of P10 animals is $0.76 \pm 0.51 \mathrm{nA}(n=5)$, compared with $6.5 \pm 0.9 \mathrm{nA}$ from P14 animals $(n=6)$, and $10.3 \pm 1.3 \mathrm{nA}(n=5)$ from P20 animals.

In line with the electrophysiology, immunostaining of developmentally matched samples with the polyclonal antibody (Fig. $3 B$ ) shows little immunoreactivity in immature IHCs (P10) and an increase in immunoreactivity from the onset of hearing (P14) to the maturation of hearing (P20). As an approximate but direct means of comparing the immunofluorescence findings with electrophysiology, the number of fluorescent puncta detected was compared with the peak BK current measured at a command potential of $40 \mathrm{mV}$ for each age normalized to the average value at P20. Figure $3 D$ summarizes the results from three separate im-
P14

P20
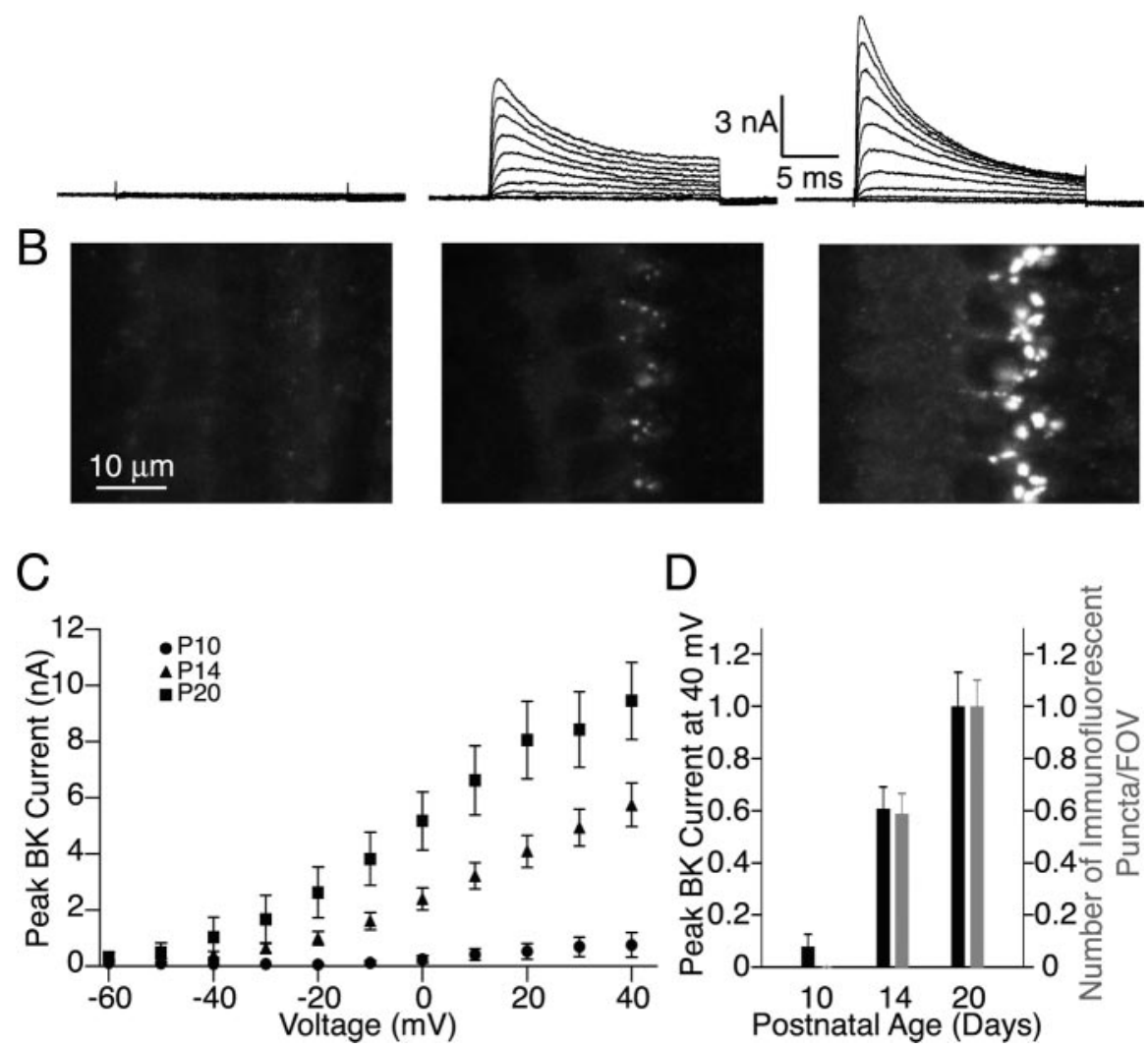

Postnatal Age (Days)

Figure 3. Electrophysiology and antibody staining reveal a corresponding developmental increase in BK current and BK channel immunoreactivity. BK currents in response to depolarizations from -60 to $40 \mathrm{mV}$ in $10 \mathrm{mV}$ steps were isolated from recordings 列 normalized to their respective values for P20 samples shows that the assessments of the developmental acquisition of the BK channel by both electrophysiology and immunoreactivity are comparable.

munostaining experiments. P10 preparations showed little BK current $(8.0 \pm 4.6 \%)$ and no immunoreactivity compared with P20 preparations. P14 preparations showed intermediate levels for both the peak BK current $(61 \pm 8.0 \%)$ and number of fluorescent puncta $(58.8 \pm 7.8 \%)$ compared with $\mathrm{P} 20$ preparations. Although not quantified, we also observed a developmental increase in the size and intensity of the fluorescent puncta. Thus, the overall patterns of developmental acquisition assessed by both electrophysiology and immunoreactivity are consistent and suggest that both methods are detecting a similar population of BK channels in mIHCs.

\section{BK channels are localized away from synapses on mouse IHCs}

The utility of immunofluorescence to detect functionally relevant populations of BK channels allowed us to investigate the subcellular localization of the BK channel in MIHCs from animals with mature hearing (P20). Although previous attempts to localize BK channels with immunostaining also report labeling of IHCs (Skinner et al., 2003), the method of secondary detection used in that study does not permit determination of the subcellular localization. We used three-dimensional reconstructions from stacks of confocal images of immunostained organs of Corti to 
A
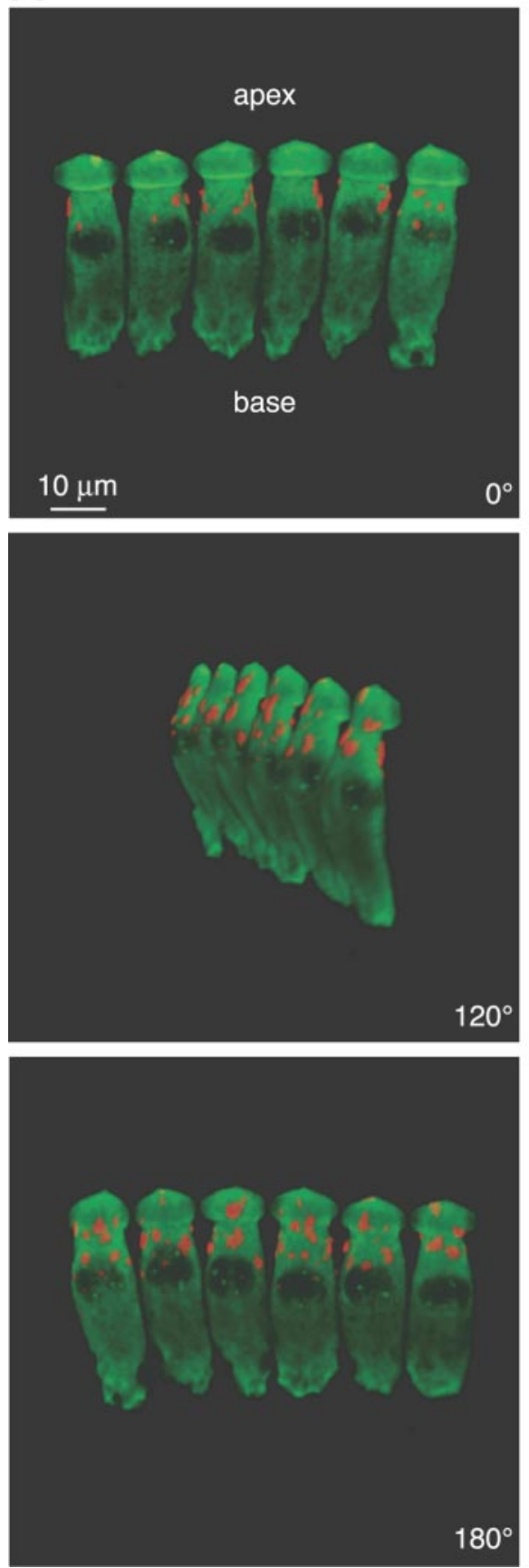

B
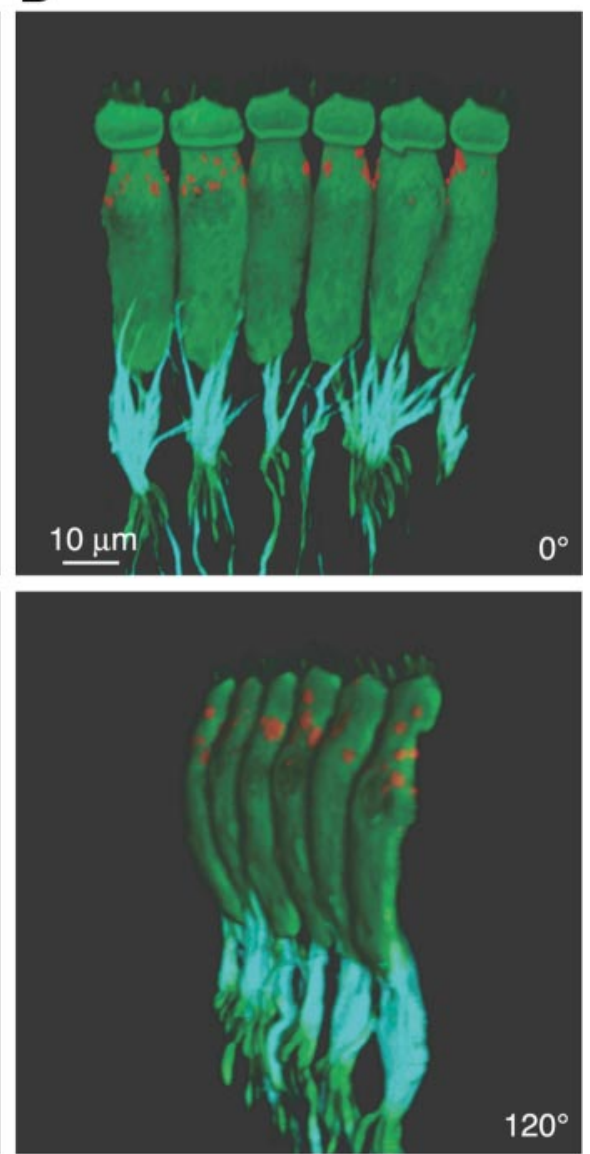

$120^{\circ}$

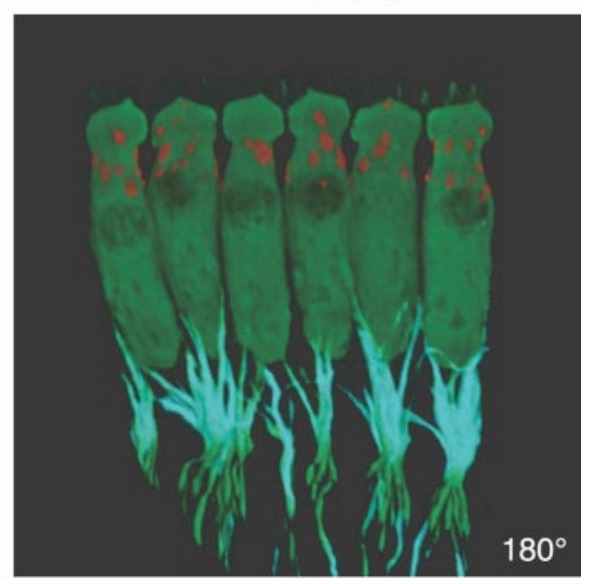

Figure 4. Antibody staining reveals extrasynaptic localization of BK channels near the apex of mIHCs. Three-dimensional reconstructions of immunostained IHCs from P20 mice were generated from stacks of confocal micrographs as described in Materials and Methods. Various angles of rotation are shown for IHCs double immunostained with either a monoclonal calretinin antibody (green), to label IHCS, and a polyclonal BK channel antibody (red; $A$ ) or IHCs triple-immunostained with a polyclonal calretinin antibody (green), monoclonal NF200 antibody (isotype $\lg G_{1} ;$ cyan), to stain afferent nerve fibers that contact the IHCS, and a monoclonal BK channel antibody (isotype $\lg _{2 A}$; red; $B$ ). Reconstructions reveal extrasynaptic localization of BK channels near the apex of mIHCs.

characterize the localization of the BK channels within mIHCs and relative to the afferent fiber projections. Figure $4 A$ shows IHCs double stained with a monoclonal calretinin antibody (green) to mark the cell body and the polyclonal BK channel antibody (red) to localize the BK channels. A number of representative IHCs are shown at different angles of rotation with their apical poles upward. As expected for a membrane-bound protein, the reconstructions show expression of the BK channel on

the surface of IHC (with the cytosol defined by the calretinin stain). However, to our surprise, the reconstructions clearly show that BK channels are located near the apex of the IHC and not at the base where synaptic sites (active zones) are expected to be present. Moreover, BK channels appear to be more densely expressed on the modiolar side of the IHC $\left(\sim 180^{\circ}\right.$ rotation), although this result was not examined further. To more directly compare the localization of the $\mathrm{BK}$ channels relative to synaptic sites, we triple immunostained mouse organs of Corti with a polyclonal calretinin antibody (green) to mark the cell body, the monoclonal BK channel antibody (red) to localize the BK channels, as well as a monoclonal antibody to the 200 $\mathrm{kDa}$ isoform of neurofilament (cyan), a protein enriched in the afferent fiber projections from the spiral ganglion that synapse onto IHC active zones (Berglund and Ryugo, 1991). Figure $4 B$ shows a representative number of IHCs oriented as described for Figure $4 A$. Results from the triple-labeling experiment corroborate two key findings from the double-labeling experiment. First, staining by the monoclonal antibody against the BK channel shows a similar surface and subcellular localization of the BK channels as staining by the polyclonal antibody. Second, the BK channels are not colocalized with the afferent fibers. Rotations of $360^{\circ}$ of the threedimensional reconstructions are provided on-line as supplementary information in the form of QuickTime movies (supplemental material, available at www.jneurosci.org). Our results demonstrate a significant population of apical, nonsynaptic BK channels. We detected no synaptically localized BK channels. Although we cannot exclude the possibility that specific BK channel splice variants not detected by either the monoclonal or polyclonal antibody are present in active zones, the similarity in immunoreactivity of both antibodies (Fig. 2) as well as the correlation between $\mathrm{BK}$ channel immunoreactivity and BK current (Fig. 3) argue against this possibility.

\section{Discussion}

In nonmammalian preparations such as the frog, turtle, and chick, BK channels interact with voltage-gated calcium channels to electrically tune the hair cells to specific frequencies of sound (Fettiplace and Fuchs, 1999). Work on frog hair cells has suggested that BK and calcium channels colocalize at active zones (Roberts et al., 1990; Issa and Hudspeth, 1994), sites where the afferent projections synapse onto the hair cells. The dual dependency of BK channel activation and synaptic release on calcium influx may underlie the colocalization of BK and calcium channels at sites of synaptic release in these prepa- 
rations. This colocalization may, in turn, allow more refined synaptic transmission in response to auditory stimuli. Synaptic release in mammalian preparations is also dependent on the influx of calcium from voltage-gated calcium channels (Moser and Beutner, 2000). By extrapolation from experiments done in nonmammalian preparations, BK channels have been expected to colocalize with calcium channels at active zones (Fuchs et al., 2003). However, in contrast to nonmammalian preparations, mammalian IHCs do not use an electrical tuning mechanism and are instead mechanically tuned to specific frequencies of sound by accessory structures of the cochlea. Moreover, previous work has suggested that, in marked contrast to nonmammalian preparations, BK channel activation in mammalian IHCs is not dependent on calcium influx from voltage-gated calcium channels (Kros and Crawford, 1990) and, from more recent work, is more likely dependent on calcium released from internal stores (Marcotti et al., 2004). In line with these findings, an inositol phosphate-gated internal calcium store localized to the apical pole of outer hair cells has been identified in outer hair cells (Mammano et al., 1999). Colocalization of BK channels with such a correlate in mIHCs would provide the calcium required for their activation and also explain their clustering at the apical end. Therefore, the BK channels in mammalian IHCs need not be colocalized to presumably synaptically localized calcium channels. Ultimately, this extrasynaptic localization reflects both a novel source of calcium for activation of the BK channels and a different role for these channels in the mammalian IHCs.

\section{References}

Armstrong CE, Roberts WM (2001) Rapidly inactivating and noninactivating calcium-activated potassium currents in frog saccular hair cells. J Physiol (Lond) 536:49-65.

Armstrong CM, Bezanilla F (1977) Inactivation of the sodium channel. II. Gating current experiments. J Gen Physiol 70:567-590.

Bekele-Arcuri Z, Matos MF, Manganas L, Strassle BW, Monaghan MM, Rhodes KJ, Trimmer JS (1996) Generation and characterization of subtype-specific monoclonal antibodies to $\mathrm{K}+$ channel $\alpha$ - and $\beta$-subunit polypeptides. Neuropharmacology 35:851-865.

Berglund AM, Ryugo DK (1991) Neurofilament antibodies and spiral ganglion neurons of the mammalian cochlea. J Comp Neurol 306:393-408.

Fettiplace R, Fuchs PA (1999) Mechanisms of hair cell tuning. Annu Rev Physiol 61:809-834.

Fuchs PA, Glowatzki E, Moser T (2003) The afferent synapse of cochlear hair cells. Curr Opin Neurobiol 13:452-458.

Galvez A, Gimenez-Gallego G, Reuben JP, Roy-Contancin L, Feigenbaum P, Kaczorowski GJ, Garcia ML (1990) Purification and characterization of a unique, potent, peptidyl probe for the high conductance calciumactivated potassium channel from venom of the scorpion Buthus tamulus. J Biol Chem 265:11083-11090.

Issa NP, Hudspeth AJ (1994) Clustering of $\mathrm{Ca}^{2+}$ channels and $\mathrm{Ca}^{2+}$. activated $\mathrm{K}+$ channels at fluorescently labeled presynaptic active zones of hair cells. Proc Natl Acad Sci USA 91:7578-7582.

Kimitsuki T, Kawano K, Matsuda K, Haruta A, Nakajima T, Komune S (2003) Potassium current properties in apical and basal inner hair cells from guinea-pig cochlea. Hear Res 180:85-90.

Kros CJ, Crawford AC (1990) Potassium currents in inner hair cells isolated from the guinea-pig cochlea. J Physiol (Lond) 421:263-291.

Kros CJ, Ruppersberg JP, Rüsch A (1998) Expression of a potassium current in inner hair cells during development of hearing in mice. Nature 394:281-284

Langer P, Gründer S, Rüsch A (2003) Expression of $\mathrm{Ca}^{2+}$-activated BK channel mRNA and its splice variants in the rat cochlea. J Comp Neurol 455:198-209.

Mammano F, Frolenkov GI, Lagostena L, Belyantseva IA, Kurc M, Dodane V, Colavita A, Kachar B (1999) ATP-Induced $\mathrm{Ca}^{2+}$ release in cochlear outer hair cells: localization of an inositol triphosphate-gated $\mathrm{Ca}^{2+}$ store to the base of the sensory hair bundle. J Neurosci 19:6918-6929.

Marcotti W, Johnson SL, Kros CJ (2004) Effects of intracellular stores and extracellular $\mathrm{Ca}^{2+}$ on $\mathrm{Ca}^{2+}$-activated $\mathrm{K}^{+}$currents in mature mouse inner hair cells. J Physiol (Lond) 557:613-633.

Moser T, Beutner D (2000) Kinetics of exocytosis and endocytosis at the cochlear inner hair cell afferent synapse of the mouse. Proc Natl Acad Sci USA 97:883-888.

Oliver D, Knipper M, Derst C, Fakler B (2003) Resting potential and submembrane calcium concentration of inner hair cells in the isolated mouse cochlea are set by KCNQ-type potassium channels. J Neurosci 23:2141-2149.

Roberts WM, Jacobs RA, Hudspeth AJ (1990) Colocalization of ion channels involved in frequency selectivity and synaptic transmission at presynaptic active zones of hair cells. J Neurosci 10:3664-3684.

Rüsch A, Ng L, Goodyear R, Oliver D, Lisoukov I, Vennstrom B, Richardson G, Kelley MW, Forrest D (2001) Retardation of cochlear maturation and impaired hair cell function caused by deletion of all known thyroid hormone receptors. J Neurosci 21:9792-9800.

Sanchez M, McManus OB (1996) Paxilline inhibition of the $\alpha$-subunit of the high-conductance calcium-activated potassium channel. Neuropharmacology 35:963-968.

Shnerson A, Pujol R (1981) Age-related changes in the C57BL/6J mouse cochlea. I. Physiological findings. Brain Res 254:65-75.

Skinner LJ, Enee V, Beurg M, Jung HH, Ryan AF, Hafidi A, Aran JM, Dulon D (2003) Contribution of $\mathrm{BK} \mathrm{Ca}^{2+}$-activated $\mathrm{K}^{+}$channels to auditory neurotransmission in the guinea pig cochlea. J Neurophysiol 90:320-332.

Zheng JL, Gao WQ (1997) Analysis of rat vestibular hair cell development and regeneration using calretinin as an early marker. J Neurosci 17:82708282 . 